may get more thorough treatment than the left eye, even from him. In a room a baby is always left lying in its crib in the same position, picked up in the same manner, and the mother or nurse is likely to begin always with the same eye in the treatment, to the detriment of the other eye.

The remedy is, obviously, to instruct the woman to begin carefully with the right eye, for instance, at one treatment and the left eye at the next treatment. When an ulcer is present on one eye she should favor that eye by beginning with it.

In opening a baby's eyes a woman is likely to place the end of her finger too far from the lid margin, or to try to open the lids while they are wet and slippery externally; or a second woman attempts to help, and stretches the lids more tightly shut by keeping too far from the edge.

What is said here of ophthalmia neonatorum holds true also, of course, for infectious ophthalmias in all children up to an age when the two eyes can readily be opened equally.

2228 South Broad Street.

\section{A SIMPLE METHOD FOR REMARKING THE SQUARES IN THE COUNTING-CHAMBER OF A ZEISS HEMATOCYTOMETER.}

\section{PATRICK MOLONEY, L.R.C.P., L.R.C.S. CAPE COLONY, SOUTH AFRICA}

The inexperienced blood counter sometimes has difficulty in cleaning the slide and counting-chamber of the Zeiss hematocytometer and often resorts to the use of alcohol and ether with disastrous results. These agents remove the color from the demarcating lines of the various squares and the instrument is rendered useless. Some laboratory authorities have not been able to suggest anything better than mubbing the cell with a soft lead pencil and polishing with a soft linen cloth, or they advise the return of the slide to the maker for the purpose of remarking. Having noticed that the glass beads in the mixing-chamber had a strong affinity for Toison's fluid, ${ }^{1}$ I determined to rub a piece of gauze moistened in this solution gently over the countingchamber. The result was excellent, the boundaries of the squares being clearly defined, and, moreover, they retain the stain far better than that given by the application of the lead pencil.

\section{A SIMPLE METHOD OF BLOOD TRANSFUSION WITHOUT CANULA}

JOHN A. HARTWELL, M.D.

Attending Surgeon to Bellevue Hospital, Assistant Surgeon to Presbyterian Hospital, Consulting Surgeon to Lincoln Hospital NEW YORK

The operation of direct transfusion of blood has, through the work of Carrel, Crile and others, assumed the importance of a valuable therapeutic agent during recent years. Its general applicability has, however, been considerably restricted owing to its difficult tech-

1. Toison's mixture, a solution for diluting the blood in counting the red corpuscles, consists of $8 \mathrm{gm}$. of sodium sulphate 1 gm. and 160 c.c. of distilled water. nic. The suture of the vessels requires a marked degree of skill, and even in the hands of men more or less experienced in doing it often fails to give a free flow of blood. The use of the canula, while not quite so difficult, is still an operation that will frequently fail in the hands of any surgeon who has not had an extensive experience in using it in animals. It has the further disadvantage of very materially lessening the caliber of the vessel where it is cuffed back over the canula. The procedure described below is almost as simple as a saline intravenous infusion and del'vers into the vein a full flow of blood, which, however, can be easily controlled if it be too great.

The artery and vein are dissected out, temporarily clamped and divided in the usual manner and with the usual care in securing the small branches. The adventitia is removed from each, but a small coil of it is left curled up on the outside of the artery about one and a half inches from the cut proximal end. Three guiding sutures of fine silk are then passed by means of a fine needle - an ordinary intestinal needle and zero silk are sufficiently fine-at intervals of 120 degrees in the circumference of the cut end of the vein. The end of the artery is greased with melted, sterilized petrolatum. The mouth of the vein is drawn open with the sutures and the artery passed directly into it for a distance of one inch. One of the guiding sutures is then passed through the rolled-up adventitia on the artery to hold the two vessels in contact, and the greater or less amount of surplus circumference of the vein is clamped or sutured so as simply to approximate the artery but not to constrict it. The obstructing clamps are removed and the blood current allowed to flow. This procedure has been tried with entire success on dogs in the Cornell Physiological Laboratory, and there is no tendency whatever to clotting of the blood. It has been used but once on the human subject (in a case of severe anemia which I saw with Dr. Freeman of Orange, N. J.), and was in that case equally successful. In this instance a small tributary vein was dissected up for an inch and left attached to the receiving vein about one inch from the anastomosis in order to determine the actual flow through the latter. When the vein nroximal to this tributary was compressed and the small clamn removed from the latter a full-sized flow of blood would be thrown through it in arterial pulsations for a dis. tance of two or three feet. Digital pressure on the artery controlled accurately the rapidity of the flow. In this way it was possible to continue the flow for fifty minutes before the donor began to show sufficient loss of blood to indicate the necessity of stopping it. $\mathrm{N}_{0}$ diminution of the stream took place at the end of that time except such as was incident to the following blond pressure. There was no back leakage around the artery. After about fifteen minutes the two vessels had become thoroughly glued together, so that even the removal of the vein-constricting clamp was possible without back leakage.

There is no difficulty experienced in inserting the artery into the vein, because the wall of the former is sufficiently rigid to make it easily enter for an inch or more without kinking. For ease of manipulation, three or four inches of each vessel are needed. Whether the flow of blood could be stopped completely by pressure on the artery for a considerable time, without clotting at the end of the inserted artery, it is impos. sible to say with our present experience. With care, 
however, complete stoppage is at no time necessary, as the flow can be thoroughly controlled, if indications arise, without actually stopping it. In case, however, for any reason a clot should form it is only the work of a minute or two to separate the vessels, remove the clot and make a re-anastomosis, and this is done without sacrificing any of the vessel length. The danger of a clot washing away in the blood stream to form an embolus is neither greater nor less than in any other method. If the cut end is coated with petrolatum no fibrin threads seem to form about it. A further inprovement may be possible by simply placing the artery end in a lateral slit in the vein wall, after tying the vein distally, but no opportunity of doing this has yet been offered on the human subject. On dogs it is easy of performance, and in these animals the artery is far more flexible than in man.

Note: All the dogs used in the experiment were fully anesthetized with ether.

50 East Fifty-Third Street.

\section{Therapeutics}

\section{COJDS}

\section{(Continued from page 213)}

While we now so frequently and constantly inveigh against the use of coal-tar products because they have been too much used by the profession in prolonged acute disease, and by the laity in repetitions of acute pain, it should not be forgotten that two or three doses of a coaltar product in a condition like an acute cold is of great advantage, not only in reducing the temperature, relieving the tensity of the circulation, dilating the peripheral blood vessels, and causing sweating, but also as an analgesic. A combination such as either of the following is efficient in shortening the first stage of acute cold and perhaps aiding in preventing its continuance.

$\mathbf{R}$

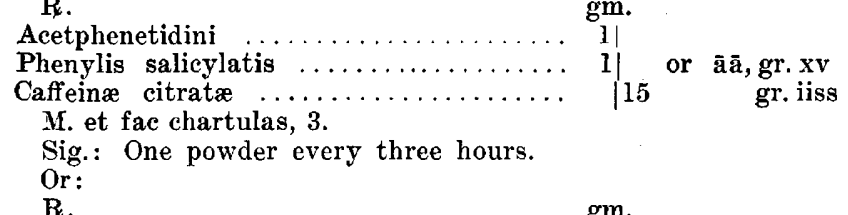

Morphinæ sulphatis $\ldots \ldots \ldots \ldots \ldots \ldots$ gm

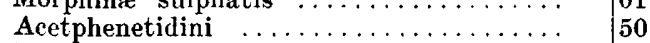

Phenylis salicylatis ...............

M. et fac chartulam, 1.

Sig.: Take at once.

As Dover's powder often causes some nausea, any combination of the desired dose of morphin with an acetanilid combination as the compound acetanilid powder, in dose deemed efficient, will effect the object desired as well as will Dover's powder.

The action of the nitrites in dilating the peripheral blood vessels and promoting sweating, when other methods are also used to cause that condition, is satisfactory. How well the sweet spirit of niter acts as a diaphoretic depends on how warm the patient is kept and how much hot water he drinks.

While we advise the drinking of hot water to promote perspiration after hot baths, etc., it is not advisable to have the patient drink much water if sweating is not to be promoted, as it fills up the blcod vessels and increases the local congestions. Hence, if it is deemed best to dilate the blood vessels and relieve congestion with aconite or by purging and not use any method to cause sweating, the amount of liquids ingested should be kept low during this stage of the cold.

The administration of aconite in small doses frequently repeated is still used by some physicians as the best treatment in the first stage of a cold, but not nearly so frequently as it used to be used, at least not alone. In the combination of a "rhinitis" tablet, which generally contains several ingredients, aconite or aconitin is often administered by the profession, and often taken by the laity without such advice. The activity of the rhinitis tablet is due to its aconite and to its atropin, and, perhaps, slightly to the small amount of morphin that it contains. A patient who is put thoroughly under the influence of aconite to abort his cold will subsequently have more prostration than with any of the above advised methods of treatment.

In any congestion of the upper air tract, throat, or nose it is certainly advisable to unload the alimentary canal, and with the method that will produce the least general discomfort and act the quickest. The cathartic selected should depend upon the time of day at which the patient is first seen. If during the daytime, a saline cathartic should ordinarily be selected; if at night, calomel is best in the dose deemed sufficient, generally perhaps best combined with bicarbonate of soda, as:

R.
Hydrargyri chIoridi mitis........... gm. $/ 20$ or gr. iii

Sodii bicarbonatis ...............

$M$, et fac chartulam, 1 .

Sig.: To be taken at once.

Or one or two grains of calomel may be given with an aloin, strychnin and belladonna tablet at bedtime. If the patient has a tendency to constipation it is advisable to give a saline, as a seidlitz powder or citrate of magnesium, or some cathartic water, in the morning.

It is often advised to give calomel in small doses, as perhaps $1 / 10$ of a grain, every half hour until one grain has been taken, aiming perhaps at the old antiphlogistic (prevention of inflammation) theory. This seems hardly advisable except as it may keep the patient interested and busy, as what is needed is a calomel movement and depletion, and not a troublesome diarrhea, which often occurs when calomel is thus administered. Also, patients susceptible to calomel may be salivated by small doses when they are not affected by a single large dose.

The abortive treatment of colds with so-called rhinitis tablets, while perhaps the best treatment when the patient must be exposed to further chilling or more dust and must keep at his occupation, seems theoretically inadvisable in the first stage of nose and throat inflammation. The main action of these tablets is the atropin action, unless the patient is somewhat prostrated by the aconite or aconitin that they contain, hence the drying up of the mucous secretions of the congested nose and throat by atropin should theoretically tend to prolong the first stage and perhaps thus prolong the second stage, as the free exudate from the inflamed mucous membrane is Nature's best method of depletion of these congested areas. Therefore, a patient housed and willing to give from ten to fifteen hours to the best abortive treatment of an acute cold should not receive rhinitis tablets, while a patient who must attend to his business may take these tablets, but ordinarily not for more than from twenty-four to forty-eight hours. A good rhinitis combination is as follows: 